\section{MRI Evaluation of Aseptic Osteo- necrosis in Children over the Course of Hyperbaric Oxygen Therapy}

A. Scherer ${ }^{1}$, V. Engelbrecht ${ }^{1}$, B. Bernbeck ${ }^{2}$, P. May ${ }^{1}$, R. Willers ${ }^{3}$, U. Göbel ${ }^{2}$, U. Mödder ${ }^{1}$

${ }^{1}$ Institute of Diagnostic Radiology, Heinrich-Heine University, Düsseldorf

2 Department of Pediatric Hematology and Oncology, Heinrich-Heine University, Düsseldorf

${ }^{3}$ Institute of Statistics, Heinrich-Heine University, Düsseldorf
Purpose: The study aimed at MRI evaluation of aseptic osteonecrosis (AON) in children over the course of hyperbaric oxygen (HBO) therapy. Material and Methods: Retrospective analysis of 72 MRI studies in 20 children presenting with AON during chemotherapy. Two groups were differentiated: Gr. I $(n=8)$ was treated exclusively with relief of weightbearing structures, Gr. II $(n=12)$ was additionally treated with HBO therapy. The MRI examinations were evaluated by a point-score system (1 6 points) by two radiologists. Results: Gr. II initially showed more severe findings (average score: 3.4 ) in comparison to Gr. I (average score: 2.65). During the follow-up time period the average scores rose to 3.2 score-points in Gr. I and 4.1 points in Gr. II. No statistically significant difference was evident between the two groups in the course of AON. Conclusion: The majority of chemotherapy associated AON which initially present with advanced findings show a progression in MRI over their further course. HBO therapy in addition to the relief of affected weightbearing structures statistically shows no significant improvement in MRI morphology during the course of the treatment.

Key words: MRI - Hyperbaric Oxygenation - Chemotherapy Osteonecrosis

\section{Introduction}

The successful treatment of malignant diseases in children, as exemplified by lymphoblastic leukaemia (ALL), has led to long term remission rates of approximately $70 \%$ [1]. Thus, knowledge of therapy associated organ damages or late complications is becoming increasingly important. Among these organ damages, we encounter the development of aseptic osteonecrosis (AON) during or after chemotherapy. Although the incidence of AON in children with ALL and bone pain was initially found to be $1.13 \%$ in a retrospective study of conventional radiographs [2], a prospective MRI study of ALL children shows frequencies significantly higher with up to $38 \%$ [3].

In the literature various reports exist concerning the course of AON. On the one hand, most authors report a progression of AON, usually resulting in osteal collapse and joint destruction

Fortschr Röntgenstr 2000; 172: $798-801$ (c) Georg Thieme Verlag Stuttgart · New York ISSN 1438-9029
[4]. Contrarily, some authors report a significant spontaneous resolution of AON [3].

Conservative therapy approaches are scarce and usually limited to joint-relief alone. Operative measures that are indicated in the case of advanced necrosis are the core decompression, transpositional osteotomy, and prosthetic joint replacement procedures [5].

In bone diseases, HBO therapy is an established method in the treatment of relapsing chronic osteomyelitis [6]. Results reporting on the outcome of HBO therapy in chemotherapy associated AON are not available in literature so far. However, promising results do exist in reports on HBO therapy of AON in experimental animal studies [7,8]. Further positive results have been reported in the HBO treatment of radioinduced osteonecrosis in humans [9]. The aim of this study was to evaluate the effects of HBO therapy on the course of chemotherapy associated AON in children by MRI.

\section{Materials and Methods}

Patients

20 children with underlying malignancies (ALL (12), Ewing's sarcomas (2), rhabdomyosarcomas (2), AML (1), Wilm's tumour (1), T-cell-lymphoma (1), and B-cell-lymphoma (1)), received MRI as a diagnostic workup of underlying osseous pain during or after chemotherapy (average: 4.2 months) of the respective skeletal region. Minimal criteria for inclusion were evidence of osteoedema or osteonecrosis in the initial MRI and at least one follow-up MRI examination. Through follow-up examinations over a course of 3-76 months and due to multifocal osteonecrosis in some patients, a total of 72 MRI examinations in 20 patients were evaluated.

In reference to the therapy of AON, the children were allocated to two different groups (Gr. I and II): In Gr. I ( 8 children) the therapy of AON was relief of weightbearing structures alone. Gr. II ( 12 children) was additionally treated with HBO therapy. The mean age of the patients at the time of the first MRI examination was $10.9 \pm 4.4$ years in Gr. I and in Gr. II $12.3 \pm 5.5$ years. The male to female ratio was 9:11. The chemotherapy protocols were dependent upon the underlying malignancy (CoALL 5/92, CoALL 6/97, SIOP 93/01, AML-BFM 93, CWS-96-high-risk, NHLBFM 95) with an average dose of $250.3 \mathrm{mg}$ Dexamethason and $1320 \mathrm{mg}$ Prednison in Gr. I and $285.5 \mathrm{mg}$ Dexamethason and $1857 \mathrm{mg}$ Prednison in Gr. II. 


\section{Hyperbaric oxygen}

The HBO therapy in Gr. II was conducted according to the guidelines of the European Consensus Conference in Hyperbaric Medicine in 1994 in Lille/France [10]. The therapy lasted approximately 130 minutes per session for each of the 12 patients. After an initial compression phase of $10 \mathrm{~min}$. a $90 \mathrm{~min}$. period of oxygen inhalation followed by a $10 \mathrm{~min}$. decompression phase was administered. Under a total pressure of $2.5 \mathrm{bar}$ oxygen inhalation was interrupted after 30 minutes by a 10 minute "normal-air" inhalation phase. A total of 30-60 pressurised "diving"-phases (average: 43) were sequentially performed during a total time period of $6-12$ weeks (average: 8.6 weeks).

\section{Magnetic resonance imaging}

MRI was performed on a $1.0 \mathrm{~T}$ (Philips Gyroscan) and a $1.5 \mathrm{~T}$ (Siemens Magnetom Vision) scanner. Only those sequences were used in the evaluative process that were acquired in every examination in all patients alike: 1 . Coronal Short Time Inversion Recovery (STIR) (TR 1500-5300 msec, TE 15-60 msec, Thk 3.0 - 5.0 mm), 2. Axial T2 weighted Spin Echo (28503800/96-120/3.0 - 6.0) and 3. Coronal T1 weighted Spin Echo (500- 740/14-16/3.0-5.0).

The retrospective analysis was performed independently by two experienced radiologists who were blinded with respect to patient name, clinical data, date of the MRI examination and the HBO therapy. Evaluative criteria included size, number, and localisation of AON. Concerning the position of osteonecrosis, a differentiation between epi-, meta-, and diaphyseal location was noted. In the case of epiphyseal necrosis, special attention was given to the involvement of joint surfaces. A score system was used to evaluate MRI findings. The criteria of the score system are summarised in Table $\mathbf{1}$. The MRI examinations of operated patients were registered independently and were excluded from comparative statistical analyses of both groups.

Table 1: Score system for evaluation of aseptic osteonecrosis (AON) in MR imaging

\begin{tabular}{ll}
\hline Score & MRI findings \\
\hline 1 & $\begin{array}{l}\text { discreet, spotty osteoedema } \\
\text { extended osteoedema }\end{array}$ \\
3 & $\begin{array}{l}\text { osteonecroses (number }<5, \text { primarily meta- and } \\
\text { diaphyseally located) }\end{array}$ \\
4 & $\begin{array}{l}\text { osteonecroses (number }>5 \text {, partially located in the epihysis) } \\
\text { osteonecroses with joint surface involvement } \\
6\end{array}$ \\
\hline
\end{tabular}

\section{Statistic evaluation}

Bias was measured by the Wilcoxon signed-rank test. Both groups were tested for the following variables with the Wilcoxon rank-sum test: patient age, survey period, startscore, score increase, and final score. The signed-rank test was calculated to evaluate whether a significant change in score was seen between both groups. The increase in score was calculated for three periods during the survey ( $<6$ months, $6-12$ months, $>12$ months). In the case of more than one examination per interval, an average was calculated and used for boxplots. In addition, the patients were categorised according to their change in score into groups of: 1 . Improvement, 2 . Unchanged, 3 . Worsened. The groups were tested for significant changes with the chi-square test.

\section{Results}

The skeletal regions showing AON most frequently in the 20 patients were the pedal bones as well as the hip and knee joints. Fig. 1 summarises the findings in these and other regions of AON manifestation along with their frequency distribution.

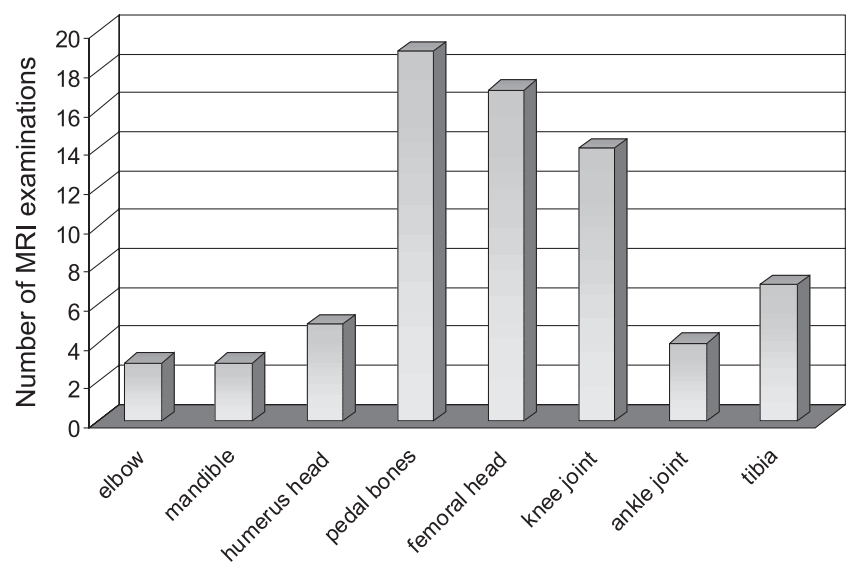

Fig. 1 Frequency distribution of aseptic osteonecrosis (AON) localisation in 72 MRI examinations from 20 children.

Initial findings revealed poorer scores with an average difference of 0.75 score-points between Gr. I (2.65 scorepoints) and Gr. II (3.4 score-points). The highest individual score in the initial MRI in Gr. II was 6 points and was one point higher than the highest score in Gr. I. The lowest initial score (2 points) was the same for both groups. If one divides the initial status into patients with osteoedema (score $\leq 2$ points) versus those with osteonecrosis (score $\geq 3$ points), then we clearly find more affected bone regions $(n=25)$ with the more beneficial finding of osteoedema in Gr. I ( 6 of 10) than compared with Gr. II ( 5 of 15). Based upon these initial findings, subsequent analyses in both groups show a statistically significant $(p<0.05)$ score increase. On average, this is $0.55 \pm 1.8$ points for Gr. I and $0.7 \pm$ 1.3 points for Gr. II (Fig. 2). Aside form these findings 3 bone regions in Gr. II showed morphologic improvement. In 5 further bone regions the findings remained unchanged. In total 8 bone regions were influenced positively by HBO therapy. 5 of these 8 bone regions initially showed findings equivalent to score values $\leq 2$ points, i.e. osteoedema. Beside the changes from the initial start-score and the patient's last examination, time dependent changes compared to the start-score were evaluated for the 3 observed intervals (Fig. 3). In addition, the average scores for the observed intervals were calculated independently from the start scores. For Gr. I, these were 2.6, 3.5, and 3.8 points and for Gr. II 4.0, 3.9, and 5.2 points. No statistically significant difference for both groups existed concerning the difference in MRI findings for the course of AON.

More children from Gr. II (10 of 12) clinically showed a regression of bone pain in comparison to Gr. I (3 of 8), 


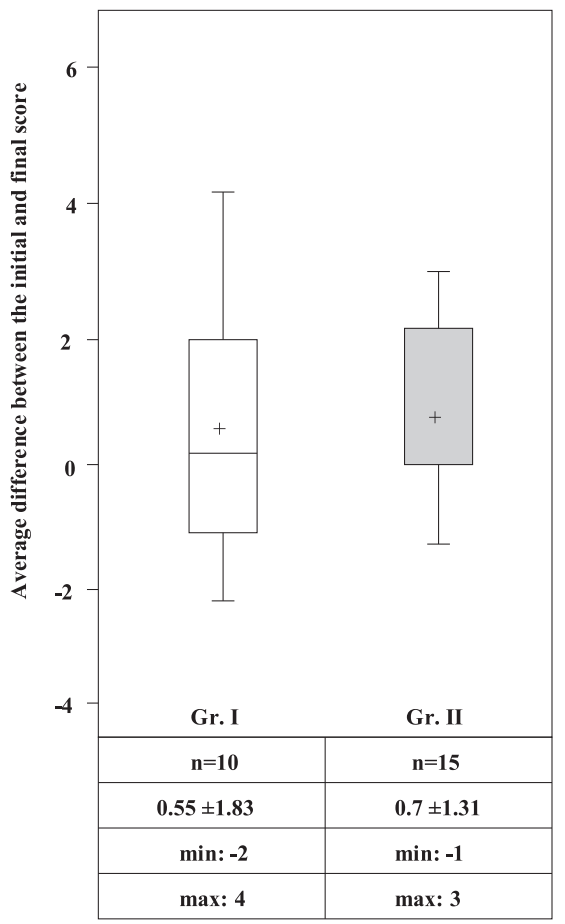

Fig. 2 Comparison of Gr. I and II in reference to the average difference between the initial and last score in the MRI examinations.
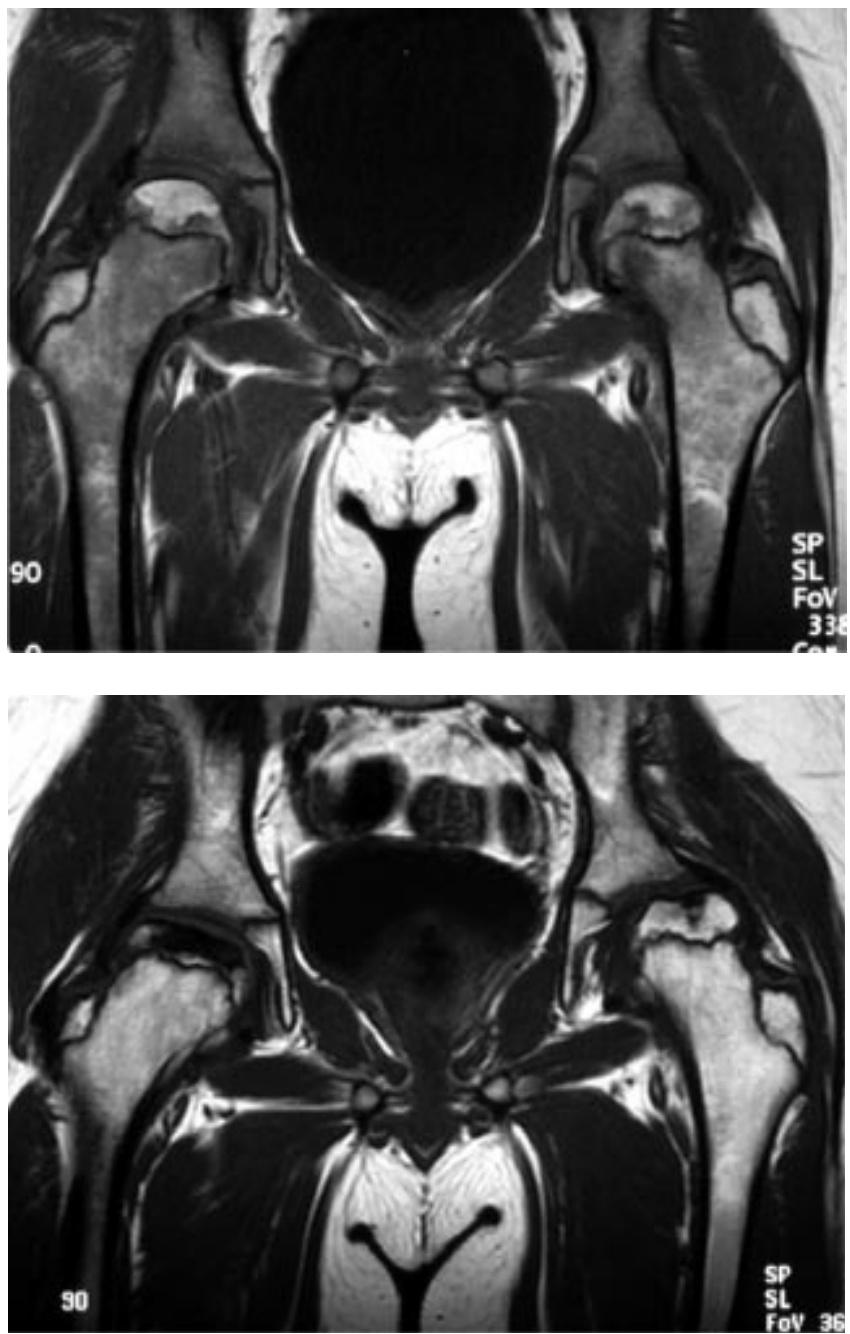

Fig. 4 Coronal T1w Spin-Echo Images (TR 500 msec, TE 14 msec, Thk $4.0 \mathrm{~mm}$ ) of the hip joint in a 11.3 year old male from Gr. II. (a) Initial finding: $A O N$ in the region of both femoral bones and femoral head epiphyses with partial contact to the joint surface (score: 5). (b) Follow up under HBO therapy one year later: Progression of findings (score: 6) with beginning left and right sided bone-height decrease and femoral head deformity.

Fig. 3 Comparison of Gr. I and II with the average difference to the start score in the course of MRI examinations relating to 3 observed time intervals ( $<6$ months, $6-12$ months, $>12$ months).

independent from MRI findings. Individual cases showed a worsening of lesion morphology (Fig. 4) despite clinical improvement. The patient compliance during HBO therapy was good. Only one patient from Gr. II discontinued HBO therapy for one month.

During the observed time period, 3 patients from Gr. II and 2 patients from Gr. I were surgically treated in the affected bone areas. In conjunction with MR findings, the surgically removed tissue-histology confirmed osteonecrosis.

\section{Discussion}

Chemotherapy associated bone pain in children leads to the differential diagnoses of therapy induced neuropathy, AON, osseous infection, and bone marrow infiltration by the underlying disease [11]. As a non-invasive diagnostic method with excellent soft-tissue contrast, MRI is the method of choice in evaluating bone marrow. In correspondence with the classification by Mitchell et al., AON shows typical findings in MR dependent on its respective stage in time [12].

The therapeutic measures available for AON are limited. Conservative measures focus on the relief of affected weightbearing joints. Since the lower extremities are usually a primary focus for AON, a relief through crutches can induce further osteonecrosis in the shoulder [13]. Among the surgical treatments, the most established are core decompression, transpositional osteotomy, and prosthetic joint replacement [5]. Resultant upon a progression of the AON, $25 \%$ of our patients were surgically treated. All of these patients showed advanced osteonecrosis with destruction of the joint surface and subsequent deformity (score: 6). 
$\mathrm{HBO}$ is a new conservative therapeutic option in the treatment of AON. During hyperbaric oxygenation therapy, the patient inhales $100 \%$ oxygen under positive atmospheric pressure conditions. The proportion of plasma solubilised oxygen increases linearly with the increase in surrounding atmospheric pressure. Due to this increase in partial pressure, oxygen can diffuse over longer distances and thus reach tissues which are cut off from the oxygen supply due to diminished perfusion or edema. Animal experiments were able to show an increase in revascularisation under HBO therapy after radioinduced osteonecrosis of the mandible. The density of vessels in the mandible was found to be 8 - 9 times higher in the HBOtreated group in comparison to the control group without $\mathrm{HBO}$ therapy [14]. In addition, beneficial effects on osteogenesis and collagen synthesis were found in animal models. Even in avascular femoral head necrosis as a non-radio-induced form of AON, significantly fewer lesions were found under HBO therapy in comparison with a control group in the animal model [7]. Similar studies in the animal model also showed faster healing rates under HBO therapy of femoral head necrosis [8]. Besides suggesting a preventive influence in the development of $\mathrm{AON}$, a supportive effect in fibro-, osteo-, and angioblastogenesis is assumed to be likely under HBO therapy. While HBO is already successful in use in the therapy and prevention of radioinduced osteonecrosis, no publications have yet described the influence of $\mathrm{HBO}$ on chemotherapy associated AON.

In our patients large epiphyseal AON close to joint surfaces (score $\geq 4$ ), more frequently resulted in a collapse of joint surfaces in both groups (Gr. I and II). In contrast, meta- and diaphyseal AON tended to show stability over the further course. Osteoedema (score $\leq 2$ ), especially under HBO therapy, showed stability or improvement over the further course ( 5 of 5 affected areas). Since the results were statistically not significant, one cannot further determine whether an improvement was resultant on HBO therapy or spontaneous and independent thereof.

\section{Conclusions}

In advanced-stage chemotherapy associated AON, HBO therapy appears to play a positive role in pain alleviation. However, no significant changes in MRI morphology are detectable in comparison to control groups treated by relief of affected weightbearing structures alone. Individual results suggest that more discrete forms of AON, especially osteoedema, can be positively influenced by HBO therapy. Thus this therapy may represent a promising conservative therapy option in the early treatment of chemotherapy associated AON in children. This hypothesis, along with the question of possible preventive effects of HBO on the development of AON, warrants further studies.

\section{Literature}

${ }^{1}$ Janka-Schaub GE, Harms D, Goebel U, et al. Randomized comparison of rotational chemotherapy in high-risk acute lymphoblastic leukaemia of childhood - follow up after 9 years. CoALL study group.Eur J Pediatr 1996: 640-648

2 Bömelburg T, von Lengerke HJ, Ritter J. Aseptic osteonecrosis in treatment of acute leukaemias. Eur J Pediatr 1989; 149: 20-23
${ }^{3}$ Ojala AE, Paakko E, Lanning P, Lanning M. Osteonecrosis during treatment of childhood acute lymphoblastic leukaemia: a prospective MRI study. Med Pediatr Oncol 1999; 32: 11 - 17

${ }^{4}$ Murphy RG, Greenberg ML. Osteonecrosis in pediatric patients with acute lymphoblastic leukaemia. Cancer 1990; 65: 17171721

${ }^{5}$ Chang CC, Greenspan A, Gershwin ME. Osteonecrosis: current perspectives on pathogenesis and treatment. Semin Arthritis Rheum 1993; 23: 47-69

${ }^{6}$ Waisman D, Shupak A, Weisz G, Melamed Y. Hyperbaric oxygen therapy in the pediatric patient: the experience of the Israel Naval Medical Institute. Pediatrics 1998; 102: E53

${ }^{7}$ Kataoka Y, Hasegawa Y, Iwata $\mathrm{H}$, et al. Effect of hyperbaric oxygenation on femoral head osteonecrosis in spontaneously hypertensive rats. Acta Orthop Scand 1992; 63: 527-530

${ }^{8}$ Levin D, Norman D, Zinman C, et al. Treatment of experimental avascular necrosis of the femoral head with hyperbaric oxygen in rats: histological evaluation of the femoral heads during the early phase of the reparative process. Exp Mol Pathol 1999; 67: 99- 108

${ }^{9}$ Ashamalla HL, Thom SR, Goldwein JW. Hyperbaric oxygen therapy for the treatment of radiation-induced sequelae in children. The University of Pennsylvania experience. Cancer 1996; 77: $2407-$ 2412

${ }^{10}$ Wattel F, Mathieu D eds.). Protocol of the 1st European Consensus Conference on Hyperbaric Medicine.Lille, France 19. 9. - 21.9.94, Reports and Recommendations

${ }^{11}$ Thornton MJ, O'Sullivan G, Williams MP, Hughes P. Avascular necrosis of bone following an intensified chemotherapy regimen including high dose steroids. Clin Radiol 1997; 52: 607-612

12 Mitchell DG, Rao VM, Dalinka MK, et al. Femoral head avascular necrosis: correlation of MR imaging, radiographic staging, radionuclide imaging, and clinical findings. Radiology 1987; 162: 709715

${ }^{13}$ Engelbrecht V, Scherer A, Bruder M, Körholz D, Mödder U. Kernspintomographie von aseptischen Osteonekrosen bei Kindern und Jugendlichen mit einer akuten lymphoblastischen Leukämie. Fortschr Röntgenstr 2000; 172: 336-341

${ }^{14}$ Marx RE, Ehler WJ, Tayapongsak P, Pierce LW. Relationship of oxygen dose to angiogenesis induction in irradiated tissue. Am J Surg 1990; 16: 519-524

\section{Axel Scherer, MD}

Institute of Diagnostic Radiology

Heinrich-Heine University

Moorenstr. 5

40225 Düsseldorf

Tel. $+49-211-8117752$

Fax + 49- 211- 8116299

E-mail: scherera@uni-duesseldorf.de 